\title{
The Development of a test bed to support the Design of Diagnostics and Prognostics Applications of a complex system
}

\author{
${ }^{1}$ Nwiwure, Don Basil Emmanuel:, ${ }^{2}$ Agbep, Nornu Steve: , \\ ${ }^{3}$ Komi, Innocent Saturday. \\ ${ }^{1,2,3}$ Department of Electrical/Electronic Engineering, Ken-saro Wiwa polytechnic PMB 20 Bori, Rivers State, \\ Nigeria.
}

\begin{abstract}
This paper presents the development of test bed to support diagnostics and prognostics applications as to enhance precision in component or subsystem. The development of this test bed is divided into three parts: the hardware, software (LabVIEW code), and the commissioning of the test bed. The hardware development involves the identification, wiring, setting, and the mounting of the components and their connections to the NI cDAQ 9172 module for proper data acquisition. The software part involves the LabVIEW code development that can be used to control the hardware component via the NI cDAQ 9172. The LabVIEW code development is carried out by developing a standard code for each of the hardware components and on the other hand the configuration of the DAQ that will be able to acquire data from these components. The test bed commissioning is about fault detection and simulation with regard to diagnostics and prognostics application using the five faults scenario which includes: clogged filter, faulty gear pump, stuck valve, leaking pipe, and clogged nozzle. The prognostic application integrates a short duration and a long duration prediction of the physical model, using the data-driven based model to obtain the remaining useful life (RUL) of the component or subsystem. However, the data driven model is utilised in this work. The diagnostic and prognostic applications are focused on how to determine the health status of component or subsystem and predict the end of life (EOL). The five faults scenarios such as clogged filter, faulty gear pump, stuck valve, leaking pipe, and clogged nozzle are the basic failure modes in many application areas which tend to reduce the fuel system efficiency. This work presents a data collection method for clogged filter, faulty gear pump, stuck valve, leaking pipe, and clogged nozzle phenomenon in the laboratory on the fuel system test rig. The different faults scenarios are being emulated by the percentage opening of the direct proportional valve (DPV). The pressure data as represented by pressure1, pressure2, pressure 3 , pressure4, pressure 5 are obtained continuously during the test period, which is represented on the graph to indicate the healthy and faulty condition. The DPV percentage opening was carried out by taking into account the pressure drop trajectory being generated from the phenomenon diagnostic test rig. The system response for healthy and faulty condition test results was plotted and used to determine the component or subsystem faults severity.
\end{abstract}

Keywords: test bed development, DPV, NI DAQ, clogging phenomenon, data-driven.

\section{Introduction}

The development of test bed facilities to support the design of the diagnostic and prognostic application of a complex system is of great concern to researchers in the recent time. The test bed fuel system which is also known as the mechatronic system comprises of mechanical, electrical, computer, and control engineering in one. The system is developed to detect, isolate and analysed the component or subsystem fault, using a graphical manner to presents information that is readable on the graphical user interface (GUI).

The development of this test bed is divided into three parts: the hardware, software (LabVIEW code), and the commissioning of the test bed. The hardware development involves the identification, wiring, setting, and the mounting of the component and it connection to NI CDAQ 9172 module for proper data acquisition. The software part involves the LabVIEW code development that can be used to control the hardware component via the NI cDAQ 9172. The LabVIEW code development is carried out by developing a standard code for each of the hardware components and on the other hand the configuration of the DAQ that will be able to acquire data from these components. The test bed commissioning is about fault detection and simulation with regard to diagnostics and prognostics application using the five faults scenario which includes: clogged filter, faulty gear pump, stuck valve, leaking pipe, and clogged nozzle.

The development of test bed to support the design of diagnostics and prognostics application is one of the ways to create the health awareness control for the system used in engineering and science application. The enhancement of safety and reliable mode of operation in a technological system such as: aircraft, land-craft, seacraft, and also in process industries, for example, refinery, and petrochemical, recycling plants. The diagnostic and prognostic application need to be considered for safety of human life and environmental protection. The 
integrated vehicle health management (IVHM) system is aimed at ensuring system safety while meeting the objective of the system which is to be considered solely on fault diagnosis and prognosis. More so, prognosis is another important part of this development which is aimed at interpreting the data obtained from diagnostic approach about the system component and used it to predict the system remaining useful life (RUL) and the end of life (EOL).

This comprehensive technology that is the IVHM, allows multiple disciplines to be integrated into the framework that enables the use of diagnosis and prognosis application in engineering, science and management. The development of test bed to support the diagnostics and prognostics application in the recent year has aided component or subsystem maintenance. The development of test bed addresses the health condition of a complex system in terms of fault and degradation. This project work is set up to simulate five different types of fault by obtaining data about the component or sub-system degradation, and use it to inform the health condition of the component or sub-system.

The test bed development carried out in the laboratory represents an unmanned aerial vehicle (UAV) fuel system that can simulate the following faults scenarios: filter clogging failure, stuck valve failure, faulty gear pump failure, leaking pipe failure, and clogged nozzle failure.

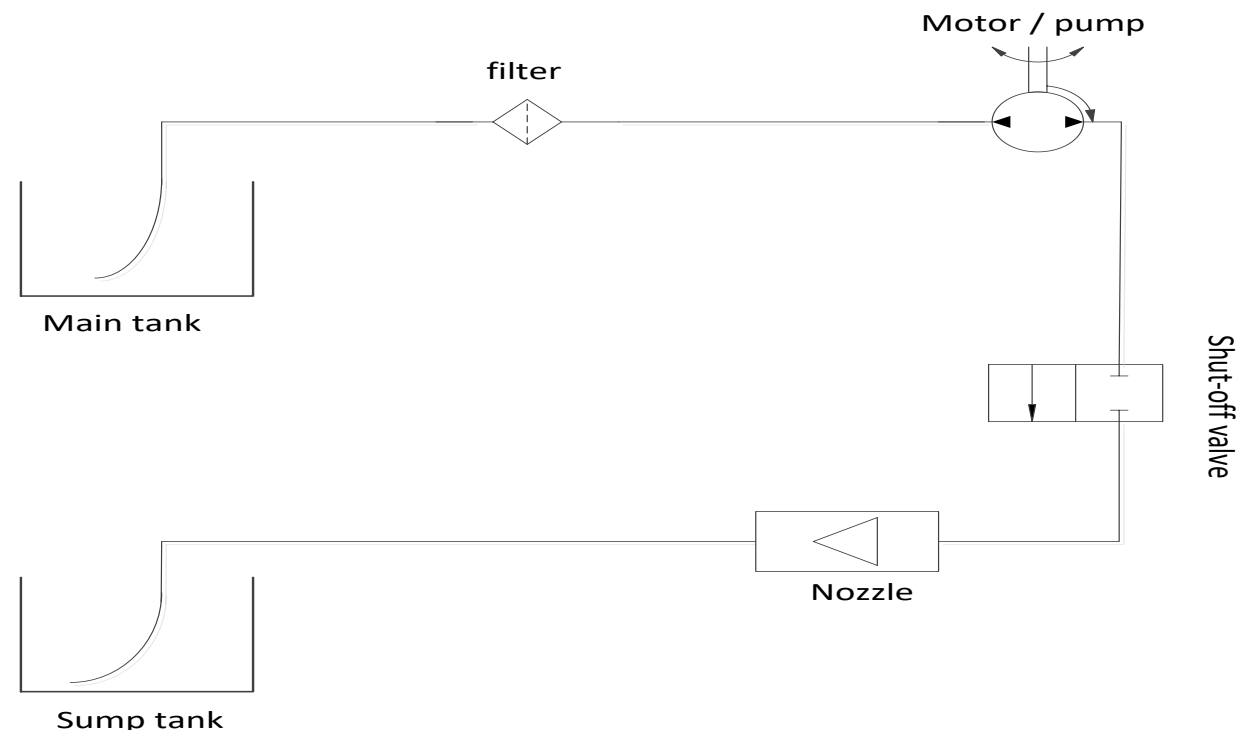

Figure1. The fuel system schematic diagram

The other aspect of this paper is sections as follows: section II deals with test bed development, while section III discussed the diagnostics and prognostics applications, then section IV is results simulation and discussion. Finally, section V concludes the paper.

\section{Test Bed Development}

This paper also give a detail explanation of the survey of literature on existing research work by various authors with regards to development of test bed, clogged filters, faulty gear pump, stuck valve, leaking pipe, and clogged nozzle, to support diagnostic and prognostic application with respect to IVHM.

The development of test bed set up in this paper work represents a UAV fuel system that can be used other related application such as aircraft, land vehicle, and watercraft. The test-bed has similar components such as power supply, tank, pump, pipes, shut-off valve, motor, power inverter, and direct proportional valves which are used for the simulation. A test-bed is known as the point or environment where transparent and a replicable test is being conducted scientifically using simulation and computational tools. The development of test bed is defined as an experimental research platform for testing of the component faults detection, and isolation using diagnostic and prognostic applications. This definition can be well implemented by developing software (LabVIEW code) that can be used to acquire data or information from the hardware component via the NI DAQ module.

[1] Asserts that test bed is developed to allowed user to safely control the UAV effectively. The development of test bed is used as a platform to investigate the component fault such as clogged filter, faulty gear pump, stuck valve, leaking pipe, and clogged nozzle [2]. The figure 2 below shows the interconnection between the test bed hardware and software via the NI DAQ module. 


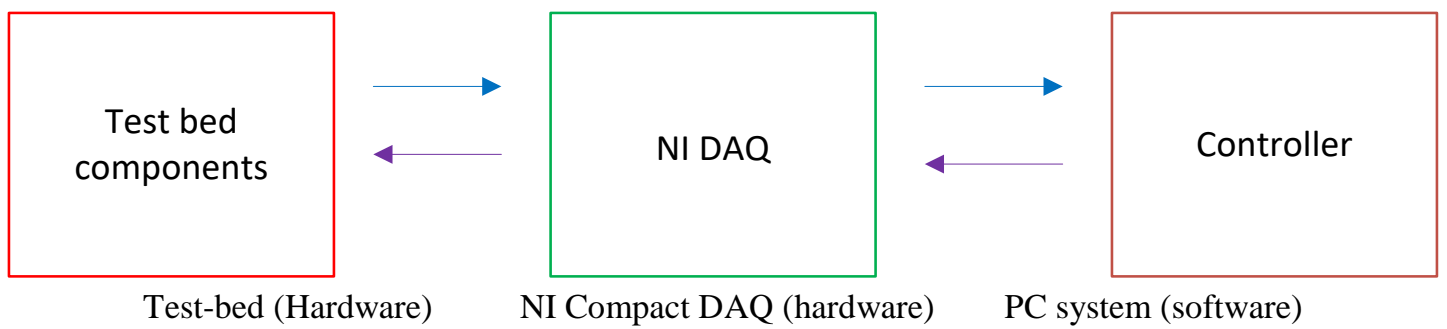

Figure 2: Test-bed hardware and software interconnection via NI DAQ module

The hardware part of the diagram in figure 2 above consist of the physical component of the test bed as mention above, where the compact DAQ modules are the point in which all component are connected as to acquire data from the respective component. Also, the PC system (software) is the environment where the LabVIEW code is developed to simulate and read the hardware component information via the GUI.

The development of this test bed to support the design of the diagnostic and prognostic application is used for fault detection and isolation as to overcome component or subsystem failure. For a system to be in perfect working condition there is the need to integrate diagnostic and prognostic application. [3] Highlight that, a better diagnostics and prognostics application reduces maintenance cost and improves system reliability as well as reducing the system life circle cost for user and producer.

The Filter is a device used for liquids filtration which in the process results to clogging known as filter clogging. This clogging is one of the faults in which this test-bed has been developed to analyse and simulates. This paper work presents the DPV as a replica of the filter and used it for the filter clogging test, as developed in the laboratory.

System failure in some cases occurs as a result of degradation during the process of operation, which is the more reason why it is necessary to obtain the degradation data of a component or subsystem and then provide information about the reliability and the remaining useful life (RUL) and the end of life (EOL). However, degradation can be obvious, which can be observed physically from a point of crack until grow to failure, while in some case, cannot be observed but by taking measurement of the component performance and ascertain it degradation process, though degradation varies from component to component [4].

Filters are designed to separate particle and water from fuel. Filter clogging that is caused by contamination is hazardous to the engine that uses fuel system. Research shows that filter clogging phenomenon occurs as a result of contamination which produces particle in fuel solvent [5]. The effect of the accumulation of particle in diesel filter was investigated and found that it reduces the filter storage capacity by several tonnes, and also affect the economy of fuel and reduces the filter lifespan [6].

\section{Diagnostics And Prognostics Applications}

The diagnostics and prognostics applications are aimed at monitoring and the detection of component or subsystem fault and then predict the component or subsystem RUL and EOL. This application is focused on the IVHM system, as to ensure that system health status complies with the standard. [7] Assert that the IVHM system has been so pronouncing and effectively used in the aircraft system. However, the IVHM system can also be used in some other vehicle such as the land vehicle, helicopters, and watercraft [8].

The diagnostics and prognostics application are introduced in the test bed to monitor the component or subsystem information and predict the future health status of the system [9].

The diagnostics application is used to determine component or subsystem parameter and identify the point of failure due to trees or degradation. This application has contributed much in terms of fault detection, isolation and a reduction in vehicle maintenance, however, the diagnostics application have been integrated into the adaptive diagnostics and prognostics test bed (ADAPT) system, for efficient diagnostics and prognostics synthesis [10].

Diagnostics and prognostics application are required in a system due to its capability to predict and manage the life cost of the system [11]. Meanwhile, this application through the PHM have improved safety in operation, increases system availability and decreases maintenance cost [12].

The diagnostics and prognostics application in aircraft operation have enhanced quality operation and improve safety as well as cost reduction in maintenance. The design of aircraft has been known as one of the complex technology that requires constant monitoring, to ascertain the vehicle health status [13].

The prognostic application is aimed at system failure prediction at the component or subsystem level, but with a good understanding of the failure occurrence, it is easy to identify and optimize the operation performance. Prognosis is regarded as fundamental within the trajectory of health management system, hence, diagnosis can detect a fault and send information to the prognosis for component health decision, which implies that prognostics application are used for system planning in case of change. There are several method used for 
system diagnosis and prognosis which include: the knowledge-based model or knowledge-based prognostic, the Fuzzy logic, hybrid models, the physic based model or model-based prognostic, and data-driven model, etc.

The knowledge based model is one of the direct applications used for component or subsystem behaviour prediction. The knowledge-based or experience based prognostics is used to determine system behaviour by using a statistical failure information obtained from the system to predict the system RUL and EOL [14].

The fuzzy logic is a mathematical framework that is used in solving an engineering problem, which can be applied on a small component or large system. This method which contains the neural network is used to provide information about the component or subsystem in terms of RUL and EOL. The fuzzy logic as extracted from the expert system approach has a based reasoning rule such as IF, THEN, ELSE was introduced by Zadeh, L.A, in 1973. However, the IF, THEN rule are used based on expert requirement [15].

The hybrid model is a combination of other prognostics approach such as the physics based or modelbased prognostics and the data-driven method. The hybrid model is introduced as to have a better prognostics approach that has the capability to control uncertainty and estimate the component or subsystem RUL and EOL accurately. The difficulties of using individual prognostics approach is eradicated by the hybrid model [16]. However, the prognostics applications are associated with physics-based model or model prognostics and datadriven approach [17].

The physics based model or the model prognostics also called the model based approaches are used explicitly to describe the physics of the component or subsystem, as to have a good understanding of the physical system degradation [18]. [19] Assert that the physics-based model applied the first principle for system prediction while the data-driven model uses the data obtained from the experiment or simulation data.

Recent research shows that there is great advanced in technology in terms of system degradation by using prognostics application to enhance effective needs for the demand of high vehicle availability, though prognostic application is seen as a support to diagnostic on the capability to determine the component or subsystem health as to predict the RUL based on degradation obtained from the vehicle operation [20]. However, the integration of diagnostics and prognostic application in engineering process maintenance, have the grate way for condition-based maintenance [21]. The RUL prediction for accuracy and precision can be measured using the Mean square error (MSE), the Root mean square error (RMSE). However, the Mean absolute deviation (MAD) and the Mean absolute percentage error (MAPE) are also commonly used.

The prognostic application as discusses above is developed to obtained information about the faulty component or subsystem from the diagnostic application and uses it to predict the component or subsystem RUL and EOL. The block diagram representation in figure 3 below shows the diagnostics and prognostics process.

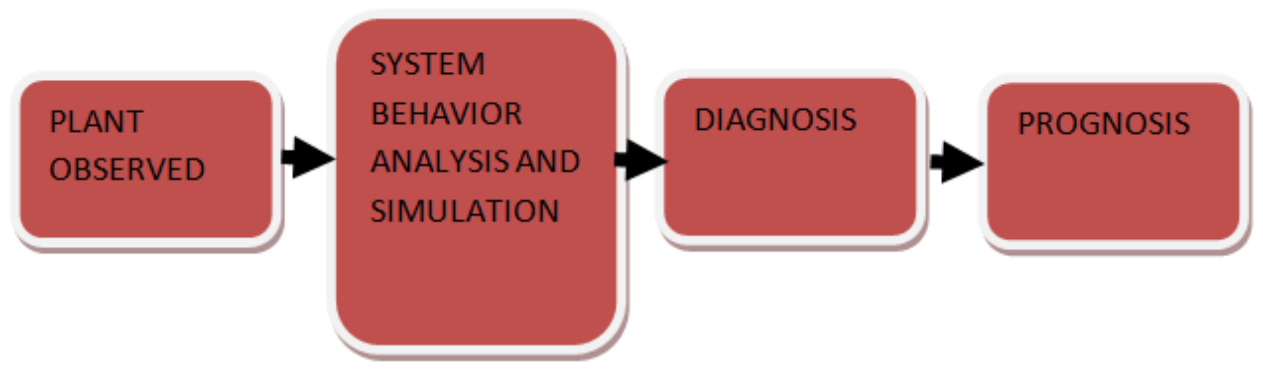

Figure3: Simple diagnostics and prognostics process

Several methods for diagnostics and prognostics applications have been discussed, but for the purpose of this paper work, the data-driven approach is used.

The data drive method is based on patterns recognition from component or sensor data. However, datadriven method for fault detection and prognostic (FDP) is recommended for a system which model are not available, that when component or subsystem vendors do not share information for a competitive reason, but where the system monitoring data is available. The data driven method assumes that the statistical feature of a system data does not change until there is a fault in the system. Also, the data-driven method gives detail information and capabilities to synthesize complex or multi-system that uses multiple sensors. The data-driven method which is based on machine method, which is considered to be the black box operation, is mostly used for fault prognostics application to learn the system behaviour from the diagnostic or condition monitoring data and interpret the state of the component or subsystem, and then predict the future failure [22].

The data-driven method evaluates the run-to-failure using the statistical-based model with the aim of putting together, the process of degradation as to predict the component or subsystem RUL. The data-driven approach uses the degradation pattern and the information obtained from the raw data displayed on the graph to estimate the failure time and predict the RUL. Prior to the advantages and disadvantages of each of these two 
methods as discussed, the data-driven is chosen for this paper work, hence it has the capability to synthesize multiple sensors in a system. This method will be employ in the data collection and analysis.

\section{Results Simulation}

The graphical representation of figure 4 and 5, and table 1 and 2 below shows the component healthy condition data. The data samples for each test conducted were plotted in every 1 minute. The results observed for the component or subsystem healthy and faulty condition test conducted on the test bed were structured as follows: (a) healthy condition test, (b) faulty condition test, (c) discussion

(a) Healthy Condition Test

The healthy condition test as discussed above was carried out and the results obtained are shown in the figure below.

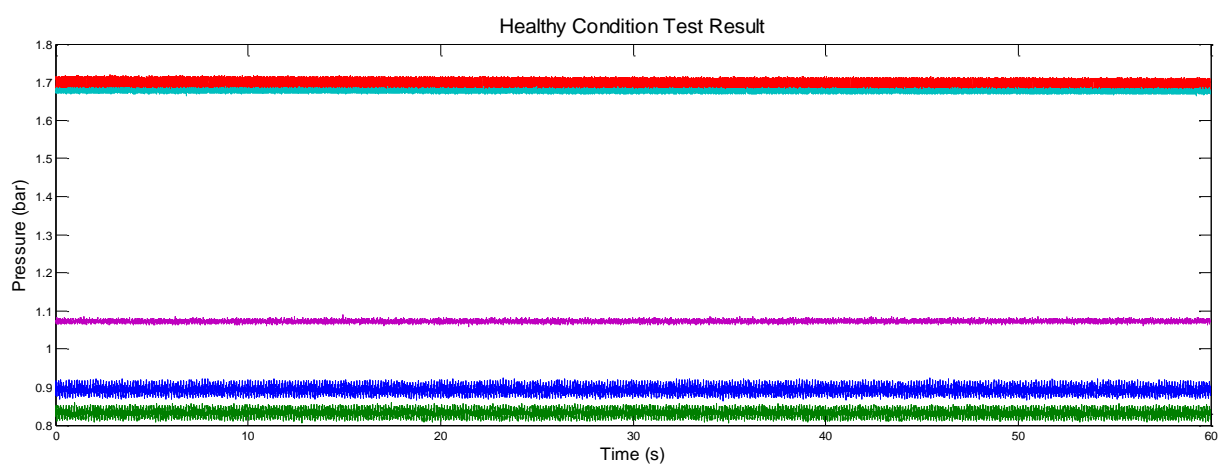

Figure 4: Healthy condition test graphical representation

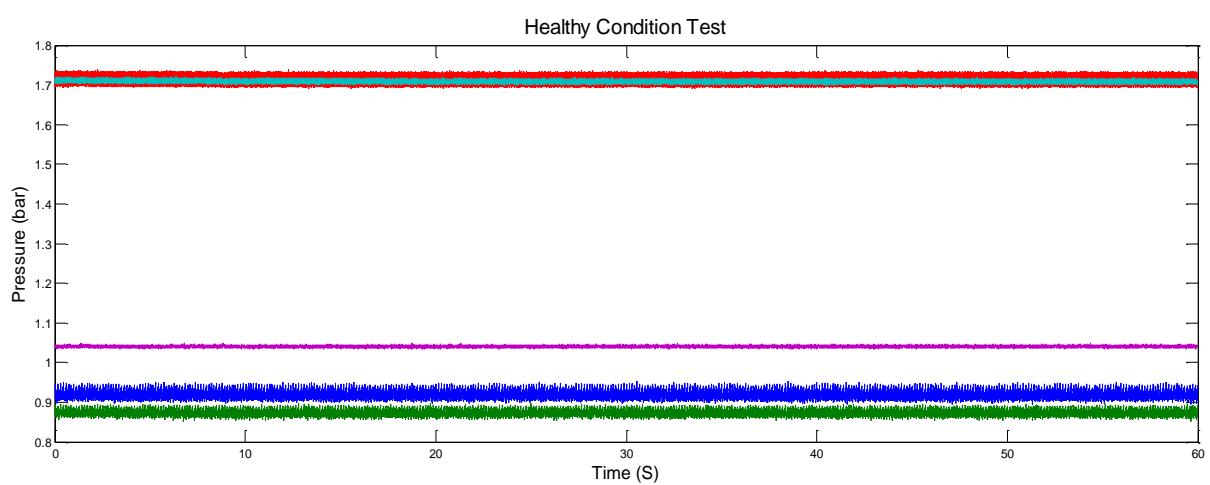

Figure 5: Healthy condition tests graphical representation

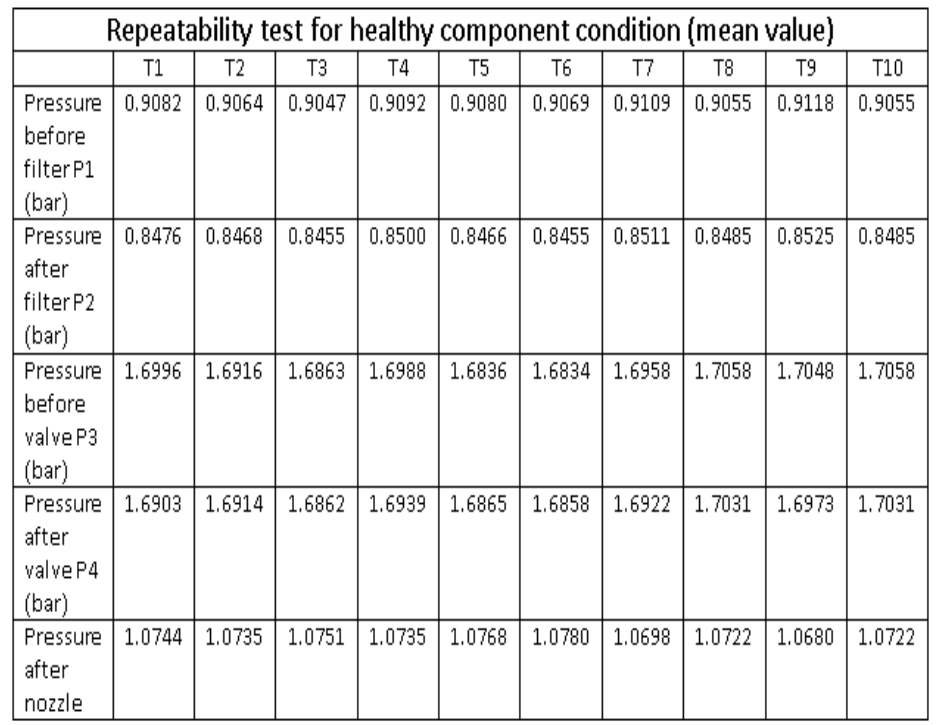

Table 1: Repeatability test for component healthy condition mean value 


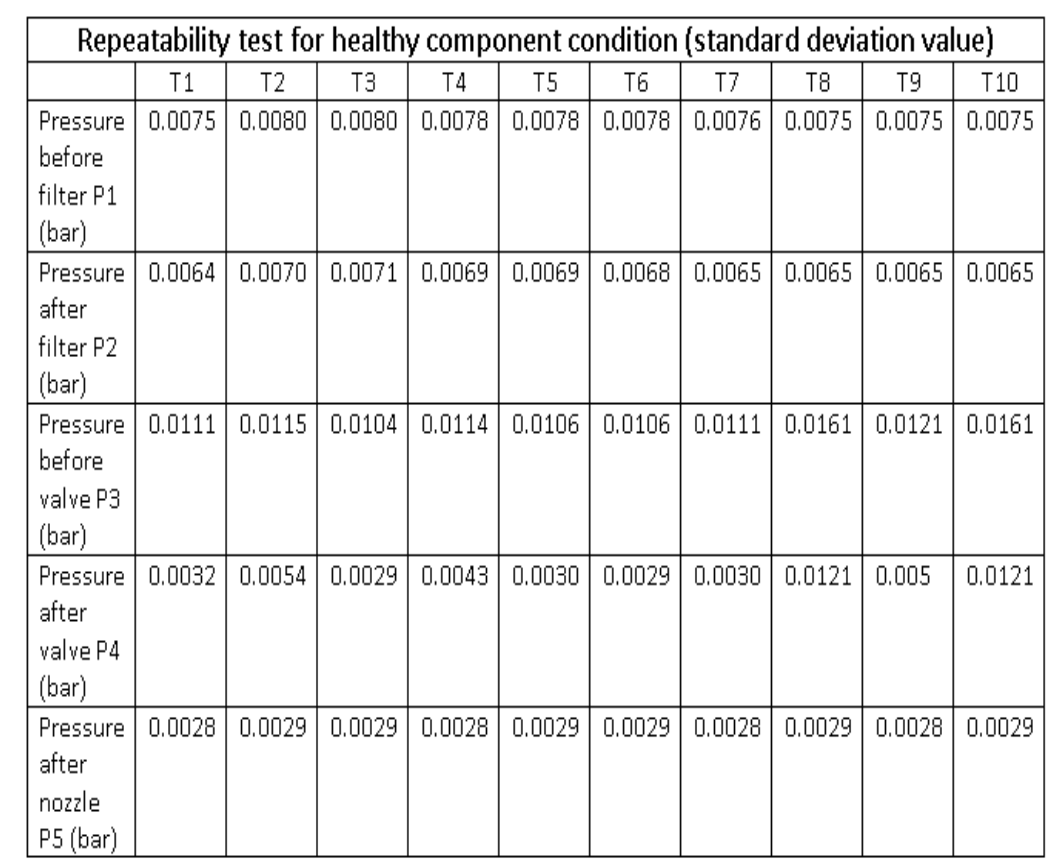

Table 2: Repeatability test for component healthy condition standard deviation value

\section{(b) Faults Condition Test}

The multiple faults condition test was carried out by percentage opening of the respective DPVs that represent the clogged filter, faulty gear pump, stuck valve, leaking pipe, and clogged nozzle. Each of these case scenarios was investigated as shown in figure 6 and 7 below.

The faulty condition case scenario was carried out by carefully injecting faults into the DPVs as it was opened from $100 \%, 80 \%, 60 \%, 40 \%, 30 \%$ for each case scenario such as clogged filter, faulty gear pump, stuck valve, leaking pipe, and clogged nozzle. The system was allowed to run for 1 minute and 60,000 samples were reads, and in every 1 second, 1000 samples were reads and write to lvm file. The data write to lvm file was then export to Matlab file where a Matlab code was developed and used to plot the response for clogged, faulty gear pump, stuck valve, leaking pipe, and clogged nozzle. The response and faults severity level for each faulty case scenario and its degradation pattern is shown in figure 6 to figure 7.

Case one: the multiple faults condition test was carried out by using the DPV1 which represents the clogged filter scenario and opened from 100\%, 80\%, 60\%, 60\%, 40\%, and 30\% while the DPV2 which represents the faulty gear pump is kept at $0 \%$, DPV3 which represents the stuck valve at $100 \%$, DPV4 which represents the leaking pipe at $0 \%$, and the DPV5 which represents the clogged nozzle at $100 \%$. The pressure before and after the filter as denoted by pressure 1 and pressure 2 were measured, also, pressure 3 and presuure 4 which denote the pressure before and after the shut-off valve were measured and then pressure 5 which is the pressure before the sump tank was measured. The graphical representation below shows the clogged filter degradation scenario.

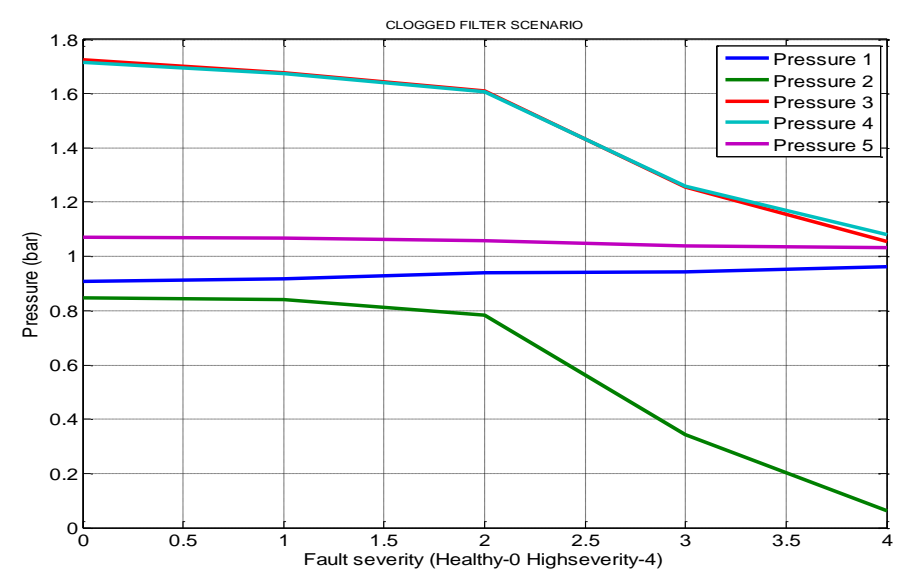

Figure 6: Clogged filter degradation scenario 
Case two: the multiple condition test for faulty gear pump represented by the DPV2 was emulated by opening the valve from $100 \%, 80 \%, 60 \%, 40 \%, 30 \%$, while the DPV1 was at $100 \%$, DPV3 at $100 \%$, DPV4 at $0 \%$, and DPV5 at $100 \%$, then pressure $(1,2,3,4,5)$ were investigated as shown in the graphical representation of figure 7 below.

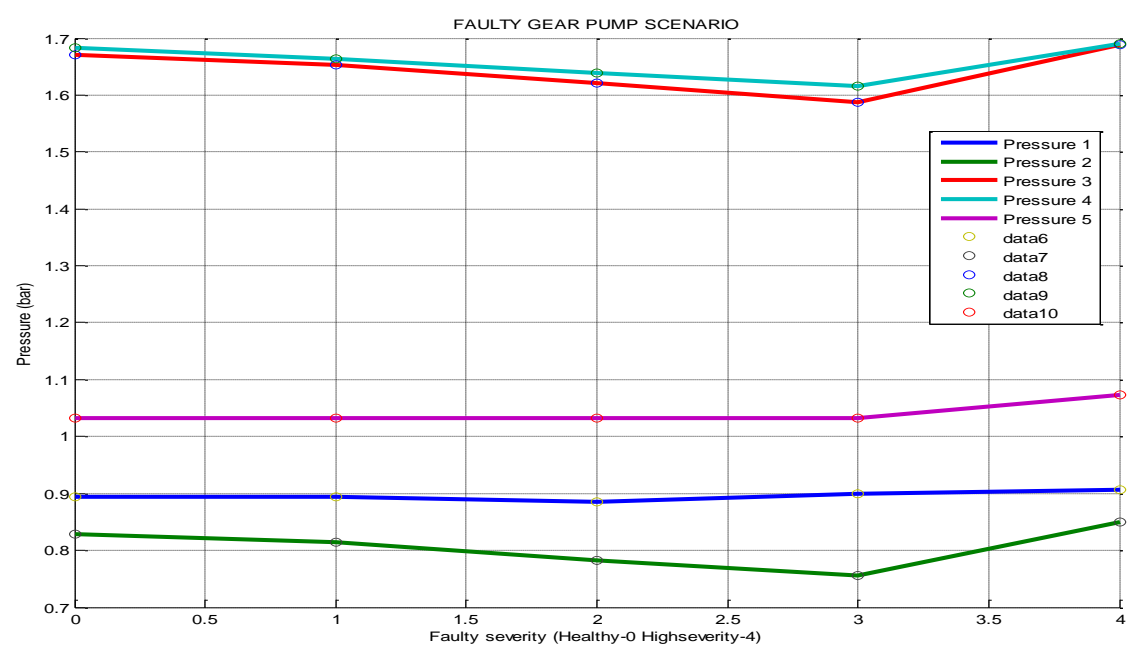

Figure 7: Faulty gear pump faults injection scenario

\section{Discussion}

The discussion section of this paper work is focused on the result obtained from the simulation and analysis of the healthy and faulty condition case scenario. The healthy condition case scenario was thoroughly investigated by setting the DPV1 at 100\%, DPV2 at 0\%, DPV3 at 100\%, DPV4 at 0\%, and DPV5 at 100\%. The pressure sensors label pressure 1 and pressure 2 were used to sense the pressure before and after the filter, then pressure 3 and pressure 4 are used to sense the pressure before and after the shut-off valve, and the pressure sensor 5 is used to sense the pressure before the sump tank.

The characteristic of the graphical representation of figure 4 and 5 shows the component or subsystem healthy condition. However, a repeatability test was also carried out to verify the healthy condition phenomenon by calculating the mean

$$
\overline{\mathbf{x}}=\sum_{\mathbf{i}}^{\mathrm{N}} \mathbf{x i} / \mathbf{n}
$$

Where $x_{i}$ is the sum of occurrence in each healthy test carried out and i ranges from $i_{1}, i_{2}, \ldots \ldots i_{n}$. And $\mathrm{n}$ is the number of measurement in the set. Also, according to the Guide to Expression of Uncertainty in measurement (GUM), the estimated standard deviation for the ten (10) tests conducted for each component or subsystem under test was calculated using the expression:

$$
\left.\mathrm{S}=\sqrt{\sum_{i=1}^{n}\left(\boldsymbol{x}_{\boldsymbol{i}}\right.}-\boldsymbol{x}_{\boldsymbol{i}}\right) / \mathrm{n}-1
$$

Where $x_{i}$ is the number of measurement, $\quad \bar{X}$ is the mean of the nth results, the type A, uncertainty evaluation is used to calculate the repeated value obtained from the ten (10) tests conducted. The estimated standard uncertainty is calculated using the formula:

$$
U=S / \sqrt{n}
$$

Where $\mathrm{U}$ is the standard uncertainty which is also called the standard deviation of the mean, $\mathrm{S}$ is the estimated standard deviation, and $\mathrm{n}$ is the number of measurement in the set.

The values obtained from the healthy condition test analysis and simulation for the ten (10) set of test (T1 - T10) were logged in every 1 minute (60) seconds. The mean value of the data for each test from T1 - T10 was calculated as presented in table 1 . The calculated mean values show that there is no significant change in pressure before and after DPV 1, but has a $0.001 \%$ drop, indicating a healthy condition. Also, the pressure before and after the valve shows the same characteristic with a drop of $0.001 \%$, similarly, the pressure after the nozzle has a similar response with a drop of $0.001 \%$. The estimated standard deviation for the ten (10) set of test was also calculated as shown in table 2 . The values obtained were less than $0.001 \%$ of the mean value. The estimated standard uncertainty values were calculated using equation 7 , and the value found to be less than $0.01 \%$. This data shows that there a constant flow through the component, which implies that the component or subsystem is in healthy condition. The mean and standard deviation of each component or subsystem under test 
is represented in table 1 and 2. The analysis and simulation results for the faulty condition test shown in figure 6 represent the clogged filter scenario and the pressure output of all the five pressure sensors are represented. Based on figure 6, the response shows that from fault severity level 2 , there is a pronounce degradation pattern indicating that the filter clogged from fault severity level 2 to 4 . Also, the result of figure 7 which represent the faulty gear pump shows that from fault severity level 1 , there is a gradual degradation pattern, indicating the faulty gear pump degradation.

\section{Conclusion}

The data-driven approach is used to perform the faults diagnosis and failure prognosis in a percentage manner. The data obtained from the faulty component or subsystem test conducted, considering the fault severity level is used to perform the fault diagnostics and failure prognostics applications. The RUL and EOL of the filter is predicted from the plot of figure 6 , where at $100 \%$ and $80 \%$ opening which correspond to the fault severity level of 0 to 1 , shows that the filter is healthy, but between $80 \%$ and $60 \%$ opening which correspond to the fault severity level of 1 to 2 , indicate the filter mid-life, similarly between $60 \%$ and $40 \%$ opening corresponding to the fault severity level of 2 to 3 shows the filter RUL and between $40 \%$ and $30 \%$ opening corresponding to the fault severity level of 3 to 4 , represents the EOL.

\section{References}

[1]. Annaz, F. (2015).UAV Test bed Training Platform development using Panda3d.Industrial Robot, 42(5), pp.450-456.doi http://dx.doi.org/10.1108/IR-01-2015-0017

[2]. Niculita, O., Skaf, Z. \& Jennions, I.K., (2014). The Application of Bayesian Change Point Detection in UAV Fuel Systems. Procedia CIRP, 22, pp.115-121. Available at: http://dx.doi.org/10.1016/j.procir.2014.07.119.

[3]. Bennett, P., Pearson, J., Martin, A., Dixon, R., Walsh, M., Khella, M. and Goodall, R. (2006).Application of diagnostic techniques for Experimental Aircraft fuel Rig. [online] http://creativecommons.org/licenses/by-nc-nd/2.5/. Available at: https://dspace.lboro.ac.uk/2134/3307 [Accessed 13 Mar. 2016].

[4]. Bennett, P., Pearson, J., Martin, A., Dixon, R., Walsh, M., Khella, M. and Goodall, R. (2006).Application of diagnostic techniques for Experimental Aircraft fuel Rig. [online] http://creativecommons.org/licenses/by-nc-nd/2.5/. Available at: https://dspace.lboro.ac.uk/2134/3307 [Accessed 13 Mar. 2016].

[5]. Roussel, N., Nguyen, T. and Coussot, P. (2007).General Probabilistic Approach to the Filtration Process.Phys. Rev. Lett., 98(11).http://dx.doi.org/10.1103/PhysRevLett.98.114502

[6]. Sappok, A., Rodriguez, R. and Wong, V. (2010).Characteristics and Effects of Lubricant Additive Chemistry on Ash Properties Impacting Diesel Particulate Filter Service Life.doi:10.4271/2010-01-1213.

[7]. Benedettini, O., Baines, T., Lightfoot, H. and Greenough, R. (2009).State-of-the-art in integrated vehicle health management. Proceedings of the Institution of Mechanical Engineers, Part G: Journal of Aerospace Engineering, 223(2), pp. 157-170.

[8]. Ofsthun, S. (2002).Integrated vehicle health management for aerospace platforms.IEEE Instrum. Meas. Mag., 5(3), pp.21-24.

[9]. Zakwan, S., Eker,, O. and Jennions, I. (2015). Towards System Prognostics: Filter Clogging of a UAV Fuel System. [online] Available at: https://www.phmsociety.org/sites/phmsociety.org/.../phmc_15_045.pdf [Accessed 13 Mar. 2016].

[10]. Deb Somnath, SudiptoGhoshal, Amit Mathur, Roshan Shrestha, and Krishna R. Pattipati.(1998). "MultisignalModelingforDiagnosis, FMECA, and Reliability."In Proceedings of the IEEE International Conference on Systems, Man, and Cybernetics. 11-14 October, 1998). doi10.1109/ICSMC.1998.725125

[11]. Byington, C., Roemer, M. and Galie, T. (2002).Prognostic enhancements to diagnostic systems for improved condition-based maintenance [military aircraft].Proceedings, IEEE Aerospace Conference, Aerospace Conference Proceedings, 2002. IEEE (Volume: 6).doi10.1109/AERO.2002.1036120

[12]. Banks, J., Reichard, K., Crow, E. and Nickell, E. (2005). How engineers can conduct cost-benefit analysis for PHM systems. 2005 IEEE Aerospace Conference, Aerospace Conference, 2005 IEEE, Big Sky, MT, 2005, pp. 3958-3967. doi: 10.1109/AERO.2005.1559701

[13]. Khan, F., Jennions, I. and Sreenuch, T. (2013).Integration Issues for Vehicle Level Distributed Diagnostic Reasoners. SAE Technical Paper Series.doi:10.4271/2013-01-2294.

[14]. Vachtsevanos, G., Lewis, L., Roemer, M., Hess, A. and Wu, B. (2006), Intelligent Fault Diagnosis and Prognosis for Engineering Syst, Wiley. http://alvarestech.com/temp/PDP2011/DiagnosticoFalhaFMEADesenvolvimentoProduto.pdf. ISBN-13: 978-0-047172999-0

[15]. Sikorska, J., Hodkiewicz, M. and MA, L. (2011).Prognostic modelling options for remaining useful life estimation by industry.Mechanical systems and signal processing, [online] 25(5), pp.1803-1836. Available at: http://cat.inist.fr/?aModele=afficheN\&cpsidt=24105440 [Accessed 10 Apr. 2016].

[16]. Peng, Y., Dong, M. and Zuo, M. (2010). Current status of machine prognostics in condition-based maintenance: a review. The International Journal of Advanced Manufacturing Technology, 50(1-4), pp.297-313.doi 10.1007/s00170-009-2482-0

[17]. Zakwan, S., Eker,, O. and Jennions, I. (2015). Towards System Prognostics: Filter Clogging of a UAV Fuel System. [online] Available at: https://www.phmsociety.org/sites/phmsociety.org/.../phmc_15_045.pdf [Accessed 13 Mar. 2016].

[18]. Pecht, M. and Jaai, R. (2010).A prognostics and health management roadmap for information and electronics-rich systems.Microelectronics Reliability, 50(3), pp.317-323. doi10.1016/j.microrel.2010.01.006

[19]. Voisin, A., Levrat, E., Cocheteux, P. and Iung, B. (2010). Generic prognosis model for proactive maintenance decision support: application to pre-industrial e-maintenance test bed. J IntellManuf, 21(2), pp.177-193.doi 10.1007/s00170-009-2482-0

[20]. Sankavaram, C., Pattipati, B., Kodali, A., Pattipati, K., Azam, M., Kumar, S. and Pecht, M. (2009).Model-Based and data-driven prognosis of automotive and electronic systems.2009 IEEE International Conference on Automation Science and Engineering, (Institute of Electrical \& Electronics Engineers (IEEE). doi10.1109/COASE.2009.5234108

[21]. Stringer, D., Sheth, P. and Allaire, P. (2009).Physics-Based modeling strategies for diagnostic and prognostic application in aerospace systems.J IntellManuf, 23(2), pp.155-162.doi 10.1007/s10845-009-0340-4

[22]. Zhang, H., Kang, R. and Pecht, M. (2009). A hybrid prognostics and health management approach for condition-based maintenance. 2009 IEEE International Conference on Industrial Engineering and Engineering Management.10.1109/IEEM.2009.5372976 